\title{
TRABAJ O Y SALUD DE LAS DOCENTES DE LA UNIVERSIDAD NACIONAL DE CÓRDOBA: USO DE MEDICAMENTOS/SUSTANCIAS LÍCITAS Y PLAN MATERNO INFANTIL ${ }^{1}$
}

\author{
Marta Susana Giacone ${ }^{2}$ \\ Maria Cristina Silva Costa ${ }^{3}$
}

Giacone MS, Costa MCS. Trabajo y salud de las docentes de la Universidad Nacional de Córdoba: uso de medicamentos/ sustancias lícitas y plan materno infantil. Rev Latino-am Enfermagem 2004 março-abril; 12(número especial):383-90.

Este trabajo tuvo como objetivo investigar condiciones de existencia, proceso de trabajo y salud y consumo de medicamentos de docentes universitarias en edad reproductiva. Además, intentó conocer uso, opiniones y sugerencias de usuarias del Plan Materno Infantil de la Obra Social Universitaria. El estudio se encaminó desde la perspectiva teóricometodológica del materialismo histórico y dialéctico, se delineó como investigación social desarrollada a través de un estudio de campo descriptivo-correlativo e estratégico en una muestra total de 50 personas. Los resultados indican presencia de condiciones de existencia y trabajo deterioradas, afectando el perfil de salud de las docentes y el consumo de medicamentos. Alto porcentaje de las entrevistadas consideró el Plan Materno Infantil muy bueno o bueno y enfatizó la necesidad de mejor información a cerca de ella.

DESCRIPTORES: proceso de trabajo y salud; salud y sociedad; utilización de medicamentos; enfermería

\section{WORK AND HEALTH OF THE CORDOBA NATIONAL UNIVERSITY TEACHERS: MEDICAMENTS/LEGAL DRUGS USE AND INFANTILE-MATERNAL PLAN}

This study aimed to investigate university teachers in reproductive age' life conditions, work and health process and medicaments use. Beside, aimed research the use, opinions and suggestions of Infantile-Maternal Plan of the University Social Assistance clients. In dialectic and historic materialism theory and method way, this is a social research developed by a strategic and correlative-descriptive fieldwork with 50 persons sample. The results showed degraded work and life conditions, affecting the teachers' health profile and medicaments use. The Infantile-Maternal Plan is good or very good and demands best information accord the teachers in high percent opinion.

DESCRIPTORS: health and work process; health and society; drug utilization; nursing

\section{TRABALHO E SAÚDE DAS DOCENTES DA UNIVERSIDADE NACIONAL DE CÓRDOBA: USO DE MEDICAMENTOS/SUBSTÂNCIAS LÍCITAS E PLANO MATERNO INFANTIL}

Este trabalho teve como objetivo investigar condições de existência, processo de trabalho e saúde e consumo de medicamentos de docentes universitárias em idade reprodutiva. Além disso, buscou conhecer uso, opiniões e sugestões de usuárias do Plano Materno Infantil da Obra Social Universitária. O estudo encaminhou-se pela perspectiva teórico-metodológica do materialismo histórico e dialético, delineou-se como investigação social desenvolvida por um estudo de campo descritivocorrelativo e estratégico, em uma mostra total de 50 pessoas. Os resultados indicam presença de condições de existência e trabalho deterioradas, afetando o perfil de saúde das docentes e o consumo de medicamentos. Alta porcentagem das entrevistadas considerou o Plano Materno Infantil bom ou muito bom e enfatizou a necessidade de melhor informação sobre ele.

DESCRITORES: processo de trabalho e saúde; saúde e sociedade; uso de medicamentos; enfermagem

\footnotetext{
${ }^{1}$ Las opiniones expresadas en este articulo son de responsabilidad exclusiva de los autores y no representan la posición de la organización donde trabajan o de su administración; ${ }^{2}$ Enfermera, Mgtr. en Salud Materno-Infantil, Profesor Adjunto en Escuela de Fonoaudiología y Jefa de Trabajos Prácticos en Escuela de Enfermería, Facultad de Ciencias Médicas de la Universidad de Córdoba, correo electrónico: antigona8@hotmail.com; ${ }^{3}$ Docente de la Escuela de Enfermería de Ribeirão Preto, de la Universidad de São Paulo, Centro Colaborador de la OMS para el desarrollo de la investigación en enfermería, correo electrónico: mccosta@eerp.usp.br
} 
INTRODUCCIÓN

Esta investigación aborda algunos aspectos de la complejidad de la relación trabajo-salud de docentes universitarias en edad reproductiva, de la Universidad Nacional de Córdoba (UNC), Argentina, desde el enfoque que sostiene que el mejoramiento de la salud colectiva debe garantizar condiciones favorables para el desarrollo de capacidades y potencialidades biológicas y psíquicas en todos los grupos humanos.

Esta proposición se fundamenta al reconocer la historicidad o carácter social de la salud enfermedad por un lado y, por otro, del trabajo docente como un proceso social y técnico complejo, para intentar dar cuenta del proceso de trabajo, del proceso salud enfermedad, de las condiciones de vida y de la articulación entre ellos, situación que aparece difusa, no sólo para la investigación sino también para el propio trabajador.

En nuestro medio, el antecedente más cercano al problema del trabajo docente y salud está en la encuesta nacional de la Confederación de Trabajadores de la Educación de Argentina (1992-93), que no incluyó al sector universitario, con antecedentes en Córdoba y Rosario. Esta última, a fines de los 90' investigó trabajo y salud en docentes universitarios.

Un primer aspecto a analizar conceptualmente fueron las condiciones económicas y sociales de la población bajo estudio, considerando que, en las ultimas décadas, la transferencia de obligaciones operadas por el Estado a las familias ha redefinido su dinámica interna, modificando las relaciones, derechos y obligaciones de sus integrantes ${ }^{(1)}$.

En un segundo momento se trató de identificar, a partir de los procesos de vida y de trabajo que configuran la práctica docente, los perfiles de salud en función de determinados procesos de desgaste y consumo de la fuerza de trabajo docente y conocer si la organización del trabajo universitario obstaculiza o dificulta el desarrollo de capacidades colectivas e individuales.

El sector universitario sufre, desde final de la década del 70' y con énfasis desde los 90', fuerte depreciación de su trabajo, marcada por continuados ajustes presupuestarios. El $55 \%$ de los docentes de la Universidad Nacional de Córdoba son de dedicación simple (10 a 12 horas de trabajo semanal) con salarios que oscilan mensualmente entre $\$ 94,59$ (US\$32) para un Profesor Titular, a $\$ 60,12$ (US\$20) para Ayudantes de $2^{2}$, cifra inferior en más del $50 \%$ a los subsidios por desempleo que el gobierno otorga a los desempleados o indigentes ${ }^{(2)}$.

Esta situación lleva a las docentes a tener que trabajar en 2 o 3 lugares o cátedras distintas, en condiciones de precariedad laboral, por las escasas posibilidades de concursar para cerca del $60 \%$ de la planta docente de la UNC, fundamentada en la escasez histórica de presupuesto.

Se suman, a las actividades del cargo, tareas vinculadas a la organización del trabajo, condiciones medio-ambientales inadecuadas para el desarrollo de las actividades docentes y de investigación, insuficiencia de recursos materiales y técnicos. Situación agravada por el aumento de la matrícula estudiantil que no se acompaña de incremento en plantel docente, ni presupuesto.

La modalidad de organización del trabajo universitario, orientada en la última década a generar recursos propios, marca a la interna de las organizaciones una inédita desigualdad en la carga de trabajo, en las condiciones en que éste se desarrolla y en los ingresos mensuales. El Programa de Incentivos Nacional y los múltiples programas sobre el que se organiza la Reforma Universitaria (Ley de Educación Superior N:24.521 sancionada en 1995) profundizan la competencia, la fragmentación en los espacios de trabajo, la tensión y desconfianza entre pares y la intensidad en la carga laboral, obligando a disputar en espacios marcados por clientelismo político, juego de influencias y mandarinato profesoral $^{(3)}$.

Se tuvieron en cuenta en la investigación que dio origen a este trabajo, el uso de medicamentos y consumo de alcohol y tabaco, dos situaciones que adquieren en la actualidad interés creciente por el aumento del consumo y de costos, también por las prácticas de automedicación en la población. La Secretaría de Programación para la Prevención y Lucha contra el Narcotráfico dio cuenta del consumo indebido de drogas en Argentina a finales de la década pasada. Pesquisa realizada por esta Secretaría informó índices preocupantes del consumo de bebidas alcohólicas, de tabaco y de medicamentos en forma ilícita. Sedantes y estimulantes son consumidos en gran proporción por la población femenina ( $55,1 \% ; 44,9 \%)$; en el $60 \%$ de los casos el consumo se inicia después de los 20 años $^{(4)}$.

En este artículo, se buscó identificar en el proceso de vida y trabajo las tendencias en el perfil del proceso salud enfermedad, que caracterizarían a las trabajadoras 
docentes de la UNC, y si éstos se vinculan al uso de medicamentos consumidos bajo prescripción y/o sin prescripción médica, sin la inclusión de demás sustancias lícitas inclusas en la investigación original, debido a limitaciones características del informe destinado a la publicación.

Conjuntamente y en contrapartida a la institución e informantes que posibilitaron la concretización de la pesquisa, se planteó una investigación estratégica de evaluación del Plan Materno Infantil (PMI) que ofrece gratuitamente a sus afiliadas la Obra Social Universitaria (DASPU). En esta instancia se integró también a una muestra representativa de trabajadoras no docentes de la Universidad, buscando conocer si se efectivizaban los objetivos y metas del PMI, así como brindar aportes y propuestas que ayuden a orientar las políticas futuras.

Organizado integralmente el trabajo permite acercar aportes y marcar tendencias en el perfil de salud enfermedad del grupo, en nivel de Universidad, Obra Social y gremio Asociación de Docentes e Investigadores de Córdoba (ADIUC) al definir propuestas y tomar decisiones.

\section{REFERENCIAL TEÓRICO Y METODOLOGÍA DE LA INVESTIGACIÓN}

Considerando cuestiones planteadas por el problema definido, el materialismo histórico y dialéctico emerge como base filosófica y método adecuados para sostener la investigación, permitiendo aprehender las relaciones entre actores sociales, enfocando el modo de producción capitalista, las fuerzas productivas (con énfasis en el trabajo) y las relaciones de producción, para considerar las contradicciones presentes en la realidad social investigada.

Delineada como una investigación social, la investigación se realizó en Córdoba, entre junio del 2002 y marzo del 2003. Se planteó un estudio de campo descriptivo-correlativo que aporte características de las condiciones de vida y de trabajo de las docentes, que podrían incidir en sus perfiles de salud y en el uso de medicamentos (incluso alcohol y tabaco en la pesquisa original). Conjuntamente, se desarrolló una investigación estratégica para verificar si se cumplen los objetivos del Plan Materno Infantil, en la evaluación de sus usuarias.

La investigación se delineó acerca de las hipótesis: las condiciones de vida y trabajo condicionan los perfiles de salud-enfermedad docentes; las características de los cambios (introducidos por la Reforma Universitaria) del proceso de trabajo favorecen condiciones adversas para el trabajo, reflejando a la tendencia al consumo de sustancias lícitas.

La población en estudio estuvo conformada por 593 mujeres en edad reproductiva que, en el año 2001 y hasta julio del 2002, hicieron uso del Plan Materno Infantil que ofrece la DASPU. Se trabajó con una muestra aleatoria polietapica representativa de los estratos de afiliadas titulares de la DASPU: docentes y no docentes.

El proceso de selección de la muestra previó escoger del padrón de afiliaciones las mujeres que hicieron uso del plan en condición de titulares, todas gestantes que dieron a la luz. La población en estudio quedó conformada por 154 docentes y 32 no docentes (integradas para la investigación estratégica) que llegaron a término en su embarazo.

Para la conformación de la muestra se tomó, aún, de los legajos, nombre, número de legajo, dirección y número de teléfono de todas las posibles participantes del trabajo. Con rigorosos procedimientos estadísticos, seleccionamos las participantes, llegando a la muestra final conformada por 40 afiliadas docentes y 10 afiliadas no docentes, estas ultimas integradas para la investigación estratégica de evaluación del PMI.

En un primer momento se utilizó fuente secundaria de recolección de datos: legajos de todas las posibles participantes de la investigación. En un segundo momento se recurrió a fuente primaria: se confeccionó un cuestionario semi-estructurado, aplicado bajo la forma de entrevista.

Las entrevistas fueron efectuadas entre julio $y$ octubre del 2002, previo acuerdo entre las partes y aprobación del Programa de Bioética de la Secretaria de Salud (DASPU). Buscaron dar cuenta de las condiciones materiales de existencia, indagó sobre la situación social familiar y personal, de formación y de trabajo, sobre estado de salud, uso de medicamentos, tabaco y alcohol. Acercaron, también, salud reproductiva (inclusa en el trabajo original) y opiniones- sugerencias destinadas a la evaluación estratégica del Plan Materno Infantil.

Recogidos los datos, se procedió al tratamiento estadístico básico de los datos, con la confección de gráficas, que aseguraron la identificación de las recurrencias, las correlaciones de estructura social, condiciones de trabajo y salud y aseguraron el tránsito 
del cuantitativo al cualitativo en el análisis. Sucesivos acercamientos analíticos permitieron perfilar las tendencias en el perfil de salud-enfermedad según condiciones de vida y de trabajo y la posible implicancia en el uso de sustancias lícitas. Se presentó un segundo informe que da cuenta del uso del PMI.

Es posible que algunos de los resultados obtenidos no correspondan con lo que sucede en otras Universidades del país y con docentes de más antigüedad en la docencia, pero las tendencias observadas seguramente serán de valor para el análisis de situaciones semejantes. Es importante registrar, aún, que la muestra posiblemente limitó el alcance del trabajo en el análisis de uso de sustancias lícitas, por tratarse de mujeres trabajadoras en edad reproductiva, de clase media, con conocimientos del daño a la salud del feto y del recién nacido que puede causar el consumo de medicamentos no prescritos (así como los efectos adversos del tabaco y el alcohol incluidos en la pesquisa original) en la situación específica en que se encontraban, etapa de amamantamiento.

\section{RESULTADOS}

Para la elaboración de este artículo, seleccionamos algunos de los datos que caracterizan las docentes en cuanto a trabajo, otros que son analizados a través la cruce de la variable edad y los que suportan la evaluación del Plan Materno Infantil.

Más de las tres cuartas partes de entrevistadas tenían entre 31 y 40 años; más de 40 años, 10\%. Tenían cursos de Post-grado o Doctorado, completo o en curso, $67.5 \%$. En cuanto a variable trabajo, casi el $40 \%$ de las entrevistadas estaban por concurso; $12.5 \%$ eran becarias o residentes, con actividad docente como carga anexa a su función; 38\% revestían en condición de interinas, con renovación anual del cargo; $10 \%$ trabajaban como contratadas, por hora o por proyecto. El $82 \%$ de las docentes tenía otro(s) trabajo(s) remunerado(s), de ellas, $80 \%$ con más de una función (cargo docente, funciones técnicos administrativas o eran becarias). El 60\% expresaran trabajar desde hacía más de 11 años; el 25\% entre 6 y 10 años.

En cuanto a cargo, tiempo de dedicación y sueldo básico mensual, sin antigüedad, que tenían las docentes al momento de la entrevista era: el $2.3 \%$, Profesor Titular, dedicación simple (10-12hs.semanales), \$94.59 (U\$ 31.53); $2.3 \%$, Prof. Asociado, dedicación simple, $\$ 89.73$ (U\$ 29.90); 11.6\% Prof. Adjunto, dedicación simple, \$80.01(U\$ 26.67); 6.9\% Prof. Adjunto, semi-dedicado (20hs. semanales), \$266.64 (U\$ 88.80); 30.23\% Jefe de Trabajos Prácticos (JTP), dedicación simple, \$71.61(U\$ 23.87); 27.91\% JTP, semi-dedicado, \$243.98 (U\$ 81.33); $2.3 \%$ JTP, dedicación exclusiva (40/45hs. semanales), \$725.32 (U\$ 241.77); 13.95\% Ayudante 1ำ, simple, \$65.86 (U\$21.95); 2.33\% Ayudante 2º semi-dedicado, \$231.18 (U\$ 77.06). 12,5\% Becarias o Residentes, dedicación exclusiva, $\$ 700$ (U\$230).

Se cruzó una variable independiente (edad) considerada con mejor peso de asociación, con las respuestas de 18 preguntas en relación con la variable, para iniciar una explicación más particularizada y obtener algunas conclusiones que merezcan estudios en mayor profundidad.

Los resultados obtenidos revelan que el momento en que coincide carrera académica con maternidad es una vez culminada la carrera de grado y cuando desarrollan la carrera de postgrado; de allí que el mayor porcentaje de hijos de entre uno y seis años se de en la etapa intermedia y que el número elegido por las parejas en la mayoría de los casos se ubique en dos hijos. El número del tercer hijo decrece hasta el $3 \%$ entre los 31 y 35 años y vuelve a aumentar después de los 36 años (23\%), incluyendo a las de más de 40 años (5\%).

La televisión aparece como principal medio de recreación e información para el 90\%. Estarían en condiciones de concretar de 1 a 2 salidas mensuales, con porcentajes bajos, solo las docentes de 31 a 40 años. La precariedad económica limitaría la posibilidad de recreación de más del 50\%, cifra que corroboran datos oficiales: el $57,5 \%$ de las familias argentinas era pobre por nivel de ingresos, en octubre de 2002, según el Instituto Nacional de Estadísticas y Censo.

No llega a 3 de cada 10, las que consideraron la situación económica familiar como buena, cifra que, entre los 36 y 40 años, supera las 4 de cada 10. El ingreso económico de la universidad era fundamental para la familia, en porcentajes que superan el $75 \%$ para todas las edades. El ingreso docente universitario, que en sólo el $15 \%$ de los casos alcanzaba los $\$ 700$, sea indispensable para la subsistencia y que en la etapa de consolidación económica de la familia, entre los 31 y 40 años, más del $60 \%$ exprese que la situación económica y familiar es 
regular, hasta muy mala, marca las prioridades de las mujeres y el deterioro, que comprometerían su recreación y participación social, elementos protectores de la salud que no reflejan las docentes.

Presentaban más diversificadas sus actividades, 6 de cada 10 mujeres entre 31 y 40 años, y 7,5 de cada 10 mayores de 40 años tenían 3 trabajos o más. Como la institución universitaria brinda a pocos las condiciones de estabilidad y salario que garanticen dedicarse exclusivamente a la docencia, es muy frecuente la práctica del ejercicio de la profesión con participación limitada, obligando a recurrir a otros empleos. Casi 3 de cada 10 mujeres hasta los 30 años y las mayores de 40 tenían una carga semanal total de trabajo remunerado que superaba las 50 horas. Si se agregan las horas de trabajo en la casa, cabe preguntarse ¿qué nivel de salud pueden tener?

Algo más del $80 \%$ del total de las docentes consideraran su trabajo docente como tensionante y desgastador. Más del 30\% lo consideraran aburrido o rutinario, debido a las actividades burocráticas, al exceso de prácticos y evaluaciones por la cantidad de alumnos, situación agravada en las escuelas dependientes de facultades, donde más del $30 \%$ de la planta docente es ad honorem. Sobre la organización del trabajo, más del $50 \%$ dijeran que no había integración entre compañeros o cátedras, percibiendo tensiones y disputas debido a carencia de elementos de trabajo y ocultamiento de la información. Los elementos de trabajo, para el $80 \%$, eran insuficientes, agregando algunas que habían adquirido el equipo docente para trabajar.

En este cuadro, no sorprenden las contradicciones en las respuestas relativas a cómo se sienten en el trabajo. Las respuestas expresan las paradojas propias del trabajo alienante, que les dificulta reflejar su situación emocional frente a un trabajo que les gusta, pero las condiciones en que se da, las desgasta. En un considerable porcentaje plantearon estar contentas o tranquilas y percibirse consecutivamente agresivas o nerviosas hasta los 40 años. Las mayores de 36 años plantearon sentirse, con mayor frecuencia, desbordadas y agotadas. Parecería que, superado los 40 años, gran parte de las mujeres se adaptó a las condiciones, buscando alejarse de tensiones, expresando naturalización de los procesos: me conecto con lo que me gusta y el resto afuera.

Las docentes de todas las edades, y en mayor porcentaje las más jóvenes, expresaron tener limitadas sus posibilidades de plantear iniciativas y tomar decisiones en el trabajo. A esto se suman, aún, las condiciones medioambientales inadecuadas del trabajo, para gran parte de ellas. Además, el $65 \%$ de las docentes perciben maltrato psíquico en el ámbito laboral y social: de compañeros $30 \%$, jefes o directivos $47.5 \%$, pareja $20 \%$, entre otros. El maltrato psíquico en ámbito laboral se evidencia en todas las edades; en el ámbito familiar, después de los 31 años.

Considerándose los datos, es posible comprender porque la evaluación positiva del trabajo, expresada en la información obtenida (les gusta la docencia, la eligen, no dejarían de hacerlo), coexiste contradictoriamente con la negativa (les desgasta, es intensivo, falta de instrumentos de trabajo y de toma de decisiones, maltrato).

El $38 \%$ de las entrevistadas dijeran tener problemas de salud. La totalidad de entrevistadas dijo sufrir algún tipo de malestar músculo-esquelético. Se consideraban bien informadas en el consumo de medicamentos (75\%) y obtenían lo que consumían bajo prescripción médica (casi el 90\%). Todavía, aparecen prácticas de automedicación en forma habitual u ocasional en el $75 \%$.

Los porcentajes más bajos en problemas de salud se evidenciaron en las mujeres de edad intermedia; expresan sufrir procesos patológicos algo más de 4 de cada 10 mujeres en los estratos de las más jóvenes, hasta los 30 años y pasados los 40 .

Todas las mayores de 40 años presentan algún problema visual, psicológico y/o psiquiátrico; 7.5 de cada 10 , trastornos gastrointestinales; 5 de cada 10 , malestares vinculados al aparato neurológico, inmunológico, cardiovascular o respiratorio. Entre los 36 y 40 años, 7.5 de cada 10 muestran algún síntoma psicológico y/o psiquiátrico; vinculado al aparato cardiovascular, neurológico y visual, 5.6 de cada 10; en proporciones inferiores expresaron algún malestar referido a aparato gastrointestinal, respiratorio, inmunológico o alimentario. De 31 a 35 años, aumentan al 100\% los malestares psíquicos y/o psiquiátricos; más de 8 de cada 10 dijeron tener algún problema visual y aumenta la proporción de síntomas que podrían vincularse a problemas neurológicos, cardiovasculares y respiratorios y que por lo general se identifican o atribuyen a situaciones de estrés sostenido. Hasta los 30 años de edad, más de 8 de cada 10 tienen algún problema visual; algo más de 7 de cada 10 manifestaron síntomas relacionados a problemas de orden 
inmunológico, psíquico y/o psiquiátrico y gastrointestinal; casi 6 de cada 10, neurológicos.

Del $75 \%$ de mujeres que expresaron automedicarse, en el grupo de edad hasta los 30 años, algo más de 4 de cada 10 tomaban analgésicos; casi 1.4 de cada 10 usaba antihistamínicos, antibióticos, gotas nasales y anti-inflamatorios. Entre los 31 y 35 años, 7 de cada 10 usaban analgésicos; 2.3 de cada 10 recurrían a terapias alternativas; 3 de cada 10 usaban antiinflamatorios y 1.5, antiespasmódicos; casi 1 de cada 10 antiácidos, antibióticos, antigripales y tranquilizantes. En el grupo de 36 a 40 años, que aparece con porcentajes más bajos de automedicación, más de 4 de cada 10 tomaban analgésicos; 1.3, de cada 10 usaba antiespasmódicos, antibióticos y anti-inflamatorios y no alcanza a 1 de cada 10 el uso de antiácidos, gotas nasales, antihistamínicos, antigripales. Cinco de cada 10 mujeres de más de 40 años usaban anti-inflamatorios y analgésicos; al consumo de laxantes, antibióticos, antiácidos y medicinas alternativas recurrían 2.5 de cada 10.

Los docentes atribuyeron sus malestares en casi $50 \%$ a problemas de orden personal: descuido en el cuidado de su cuerpo, malos hábitos en las posturas, trabajar con poca luz y procesos de envejecimiento. Más del 50\% lo atribuyó al doble trabajo y a la multifuncionalidad a la actual organización del trabajo que impacta en su estado mental y fisiológico. Un 8\% refirió el deterioro de su salud a las condiciones de inseguridad por las que atraviesa la sociedad argentina.

La evaluación del PMI incluyó aspectos relacionados a salud reproductiva, que no analizaremos en este artículo, limitándonos a focalizar algunos aspectos de la evaluación, sugerencias y propuestas de las usuarias.

El 28\% habían usado todas las prestaciones que ofrece el PMI, el $72 \%$ algunas. Expresaron falta de información adecuada respecto al uso del plan más del $20 \%$. Tenían muy buena opinión del PMI casi el 50\%; dijeran que era bueno, 44\%. Entre los servicios que consideraban conveniente mejorar, pusieron énfasis en información más precisa sobre el plan.

Propusieron: 1. instalación de vacunatorio en la DASPU, y que fueran brindadas las vacunas sin costo; 2 . que se flexibilizaran las fechas por orden de consulta, de medicamentos y de leche; 3 . prever cobertura de medicamentos con más descuento en problemas de embarazo, parto, puerperio y para enfermedades comunes en los recién nacidos, hasta el año de edad; 4. reducción de la burocracia como auditorías internas, seguir expedientes, informes; 5 . ampliar el tiempo de cobertura en leches maternizadas; 6 . órgano informativo, que enfatizara el cuidado de la salud y servicios de la obra social; 7. mejorar el modo de articulación: usuarios, servicios de atención médica y en la institución.

\section{ANÁLISIS DE LOS RESULTADOS}

Los resultados indican presencia de condiciones materiales objetivas y relaciones sociales de producción deteriorantes. Una era de crisis y cambios estructurales ha producido su impacto en la salud en general y en las mujeres particularmente.

La cuestión familiar limita y exige en exceso a las mujeres: su desarrollo profesional y académico está atravesado por la presencia de los hijos. Se observa, en todas las edades, dificultad para poder reflejar las condiciones objetivas de existencia expresadas subjetivamente en variedad de malestares y que ha futuro podrían establecerse como enfermedades, si no se plantean acciones de protección social. Los malestares y uso de automedicación que expresaron indicarían tendencia al deterioro en todos los grupos y prematuro en las más jóvenes, grupo que aparece como el más vulnerable. Todo esto estaría a señalar la asociación entre trabajo y salud.

Los malestares que expresaban sufrir las docentes marcan tendencias que posibilitan construir el perfil de deterioro-protección de salud-enfermedad que caracterizaría a las trabajadoras docentes en este momento histórico, que va más allá de la agregación de hechos individuales de morbilidad. No todo lo que estudiamos como deterioro expresado en lo biológico o psicológico es patológico; hay formas sutiles de destrucción-defensa que en determinadas situaciones, son capaces de producir consecuencias destructivas en la mente o el cuerpo. Abordamos la salud-enfermedad de las docentes como un proceso unitario y dinámico, configurado en la vida social, determinado tanto en los procesos generales de una clase como en lo cotidiano familiar personal donde se expresa ${ }^{(5)}$.

El sufrimiento humano que proviene de un orden injusto, con apariencia de imparcialidad económica y 
social, esconde una perversa situación para la calidad de vida. La maquinaria económica necesita garantizar su tasa de ganancia con base a la extracción de plusvalía; al no poder introducir sus productos en poblaciones empobrecidas, que no generan demanda, la salida es la baja sistemática de salarios reales que el estudio pone en evidencia ${ }^{(6-7)}$. Detrás de la reforma educativa se esconde la continuidad la misma lógica económica y de ajuste que logró extender al ámbito del conocimiento, políticas excluyentes en lo social. El gobierno nacional incumple obligaciones asumidas y las autoridades universitarias planifican los ajustes mediante reformas restrictivas, con congelamientos presupuestarios y recortes de las partidas asignadas $^{(1)}$.

El capital controla la fuerza de trabajo, entre otros mecanismos, por el sistema educativo, aplicando criterios de selección para proporcionar los recursos humanos calificados, requeridos por la formación económico social vigente. La universidad cumple su papel al ofertar una fuerza laboral heterogénea acorde los requerimientos del sistema productivo y contribuye a la devaluación de la función social de la educación, por las condiciones de trabajo que oferta.

Años de vaciamiento y devaluación de la función social de la educación llevaran a muchos docentes a considerar la docencia como complementaria de su trabajo, a otros a la crítica pasiva, a otros a buscar reacomodarse individualmente ${ }^{(3)}$. Las docentes no pueden reflejarse como trabajadoras; el profesionalismo en el que se formaron resulta particularmente importante en este tránsito del capitalismo liberal al monopólico, ideológicamente considerado como bien de intercambio, impidiendo la organización colectiva y solidaria en defensa del trabajo.

El lugar históricamente crítico de los intelectuales reformistas pareciera haber sido sustituido por el reconocimiento profesional individual y aceptación resignada de las condiciones. La necesidad de poner distancia política por parte de los pos-comprometidos enuncia que la estrategia sistemática de vaciamiento y exterminio, efectivizada desde la pasada dictadura, sigue marcando los destinos de la educación universitaria en el país.

El proyecto de desarrollo capitalista plasmado en los $80^{\prime}$ buscó la creciente calificación de la fuerza de trabajo asalariada; la sobre-calificación se observa en estas trabajadoras para posibilitar su mayor explotación, estrategia que en la universidad cobró una dimensión desconocida. Conjuntamente a este desarrollo, la educación se volvió superflua frente a la concentración monopolista y el crecimiento sostenido de la desocupación ${ }^{(8)}$. La debilidad ética observada en las concepciones expresadas a cerca de la docencia evidencia las condiciones macro-sociales que intervienen y determinan la existencia de la Universidad.

Los cargos docentes tienden a transformarse en variables de ajuste, por mecanismos como reducción de la dedicación, contrato por horas, uso de cargos en funciones impropias (docente en tareas no docentes o funcionarios con cargos docentes), situación verificada en el trabajo. La ausencia de mecanismos de evaluación docente y la falta de planes de perfeccionamiento organizados institucionalmente responden también a una concepción individual de la actividad.

Es pertinente integrar en el análisis a las consideraciones mencionadas, la división sexual del trabajo en el proceso productivo, fundamentado en la división social del trabajo. En sociedades complejas que avanzan en la especialización de funciones, se asigna a las mujeres, en ámbitos como salud y educación, para que aporte el complemento afectivo-expresivo que enriquece la acción, adoptando un carácter de práctica indispensable, pero subordinada ${ }^{(7)}$.

En el contexto de separación estructural entre actividades económicas y domésticas, que impone el modo de producción capitalista y es raíz de la marginalidad de la mujer, gaña espacio el trabajo en tiempo parcial que ofrece la universidad, con carácter subsidiario del trabajo femenino (aún que indispensable a la familia) respecto del masculino. Se observa la marginación laboral de mujeres sobre-capacitadas ocupando puestos de trabajo de baja categoría, con más de 10 años de antigüedad y escasas posibilidades de ascenso, contribuyendo a preservar las posiciones privilegiadas del varón en el orden universitario.

La universidad pública, de acuerdo a las conclusiones del estudio realizado por el Grupo Redes (proyecto Gentec de la UNESCO, 2003), se posiciona como la entidad que más lugar depara a las mujeres: "Las mujeres, más condicionadas a compatibilizar reproducción con producción, pueden ser atraídas por la modalidad de tiempo parcial que ofrecen las universidades". Esta situación se observa como relativa en la UNC, ya que el $82 \%$ de las docentes tenían otros trabajos remunerados. La docencia constituye el espacio complementario del salario, situación que atenta contra la importancia sobre las implicaciones del conocimiento que genera. 
CONSIDERACIONES FINALES

Pese a las limitaciones, las respuestas encontradas abren un abanico de situaciones que, en gran medida, reafirman las hipótesis planteadas. Los problemas, las dificultades, los obstáculos, se expresan de distinto modo en las repuestas. El trabajo intercambiable fragmentado da pie al desarrollo de procesos que desembocarán en enfermedad y se expresan en la variedad de síntomas e consumo de medicamentos que aparecieron con alta frecuencia en todas las docentes.

Los procesos relacionados al trabajo docente, a la multifuncionalidad o distintos trabajos en dos o tres instituciones diferentes, las cargas excesivas que no dan tiempo para desarrollarlas adecuadamente, las actividades pocas veces distribuidas democráticamente, la tensión de la competencia en terrenos desiguales, entre otros, producen desgaste innecesario. El trabajo se torna un puro gasto de energía en el que el producto del esfuerzo aparece ajeno y enfrentado y la actividad productiva como inútil. Las docentes, al no poder desplegar su potencialidad según el desarrollo de las fuerzas productivas, expresan fuerte tendencia a la enfermedad que incrementa el consumo de medicamentos desde edades tempranas.

Las mujeres sienten que crecen, aprenden, buscan desarrollarse. También sienten agresiones psicológicas o mentales producidas por la subvaloración de ellas como mujeres y como trabajadoras. En los espacios académicos perciben agresiones físicas variadas, adoptan por tiempos prolongados posturas que dañan su columna y articulaciones, frente a pantallas de equipos electrónicos que producen radiaciones vinculadas a trastornos oculares y músculo-esqueléticos, presentes en todas ellas.

\section{REFERENCIAS BIBLIOGRÁFICAS}

1. Vommaro PP. Reforma y Ajuste en la Universidad Argentina, presentado en el Taller Latinoamericano en Defensa de la Universidad Pública contra el Neoliberalismo. Managua; febrero 2000

2. Universidad Nacional de Córdoba. Anuario Estatístico. Córdoba (AR); 2001.

3. Vior S, Brusilovski S. La universidad argentina en los 90': políticas, procesos, propuestas, v. 1. Universidad de Laguna (Tenerife): Tempora, Segunda Época; 1998.

4. Universidad Nacional de Córdoba. Facultad de Ciencias Médicas. Escuela de Enfermería. Comisión Interamericana para el Control de Abuso de Drogas, OEA. Proyecto de enseñanza de la prevención y atención de enfermería en relación al uso indebido de drogas. Córdoba: Ed. Brujas; Octubre 1999. Serie Milenio Sin Drogas, n. 2.

Recebido em: 16.1.2004

Aprovado em: 15.3.2004
Las docentes soportan agresiones psíquicas, como la repetición de clases, de prácticos, de cientos de evaluaciones, la conducta no siempre flexible de directivos, titulares o compañeros, la información que tienen que conseguir solas, los resultados de investigación que tienen que estar en fecha. Saben además que llegarán a su casa donde las espera otra jornada, distintas demandas, con el fantasma de la aprendida sensación de culpa por no disponer de más tiempo para acompañar a la pareja y a los hijos. Todo esto, íntimamente ligado, es fuente de tensión y estrés.

Pero, soportar el progresivo deterioro de la vida impone que se va allá de la dimensión individual expresada por las docentes. Articular fuerzas es una necesidad urgente, demostrados los límites del proyecto reformista. Se impone la construcción de un proyecto solidario que marque la diferencia ético-política frente al pragmatismo que continúa proclamando una universidad de excelencia, en un país que sufre desde las últimas décadas el deterioro sostenido más profundo en las condiciones de vida y de trabajo.

\section{AGRADECIMIENTOS}

Agradecemos a todos los docentes de la Escuela de Enfermería de Ribeirão Preto y a los treinta y tres participantes de nueve países latinoamericanos que participaron en el proceso de implementación de los programas, y también al Gobierno del Japón y al Programa de Becas de la OEA por su apoyo financiero y becas que posibilitaron la implementación del "I Programa Regional de Capacitación en Investigación para Enfermeros de América Latina".

5. Laurell A. La salud de derecho social a mercancía. In: Nuevas Tendencias y Alternativas en el Sector Salud. México: Ed. Universidad Autónoma Metropolitana; 1995.

6. Braverman H. Trabajo y Capital monopolista. México: Ed. Nuestro Tiempo; 1980.

7. Castells M. O Poder da Identidade. São Paulo (SP): Paz e Terra, 1999. A era da informação: economia, sociedade e cultura, v. 2.

8. Brusilovsky S. Extensión Universitaria y Educación popular. Buenos Aires: Eudeba; 2000. 